\title{
micromachines
}

ISSN 2072-666X

www.mdpi.com/journal/micromachines

Article

\section{Multiscale Modeling of Skeletal Muscle Active Contraction in Relation to Mechanochemical Coupling of Molecular Motors ${ }^{\dagger}$}

\section{Jiangcheng Chen ${ }^{1, *}$, Xiaodong Zhang ${ }^{1, \ddagger}$, Shengmao Lin ${ }^{2,}$, He Wang ${ }^{1, \ddagger}$ and Linxia Gu ${ }^{2, \ddagger}$}

1 Key Laboratory of Education Ministry for Modern Design and Rotor-Bearing System, School of Mechanical Engineering, Xi'an Jiaotong University, Xi' an 710049, China; E-Mails: xdzhang@mail.xjtu.edu.cn (X.Z.); wh1062014896@163.com (H.W.)

2 Department of Mechanical and Materials Engineering, University of Nebraska-Lincoln, Lincoln, NE 68588-0656, USA; E-Mails: lgu@unl.edu (L.G.); linshengmao@gmail.com (S.L.)

$\dagger$ This paper is an extended version of our paper published in the 10th Annual IEEE International Conference on Nano/Micro Engineered and Molecular Systems (IEEE NEMS 2015), Xi'an, China, 7-11 April 2015.

* These authors contributed equally to this work.

* Author to whom correspondence should be addressed; E-Mail: jiangcheng.0502@163.com; Tel./Fax: +86-29-8266-3043.

Academic Editors: Wen Jung Li and Ting-Hsuan Chen

Received: 22 May 2015 / Accepted: 2 July 2015 / Published: 10 July 2015

\begin{abstract}
In this work, a mathematical model was developed to relate the mechanochemical characterizations of molecular motors with the macroscopic manifestation of muscle contraction. Non-equilibrium statistical mechanics were used to study the collective behavior of myosin molecular motors in terms of the complex conformation change and multiple chemical states in one working cycle. The stochastic evolution of molecular motor probability density distribution during the contraction of sarcomere was characterized by the Fokker-Planck Equation. Quick muscle contraction was demonstrated by the collective dynamic behavior of myosin motors, the muscle contraction force, and the muscle contraction velocity-force relation. Our results are validated against published experiments, as well as the predictions from the Hill's model. The quantitative relation between myosin molecular motors and muscle contraction provides a novel way to unravel the mechanism of force generation.
\end{abstract}


Keywords: muscle contraction; non-equilibrium statistical mechanics; molecular motor; biomechanical model; Fokker-Planck Equation

\section{Introduction}

Skeletal muscle, the impetus of human movement, is an important part of the human motor system. It generates active contractions, leading to a variety of complex and subtle movements. The biomechanics of the skeletal muscle have attracted increased attentions due to their role in regulating human movements, improving competitive sports performance, preventing sports injury, and designing effective training programs for rehabilitation.

Muscle fiber, composed of paralleled myofibrils with average diameters of approximately $1 \mu \mathrm{m}$, is the main component of individual skeletal muscle [1]. Myofibrils possess distinct patterns alternating between dark A-bands and light I-bands. The center line of light bands is defined as the Z-line, and the section between neighboring Z-lines is referred to as sarcomere, the fundamental unit of skeletal muscle. From the microscopic point of view, sarcomere contains thick and thin filaments. While the thin filament is composed of actin, tropomyosin-Tm, and troponin-Tn, the thick filament is formed by myosin molecular motors, which are key components of skeletal muscle contractions. Each thick filament is surrounded by six thin filaments and connected to the Z-line through an elastic element made of titin, whose passive elasticity plays an important role in muscle contraction.

Biomechanics research on skeletal muscles has been focused on the macro and micro levels, separately [2-5]. At the macro level, the Hill's model, based on in vitro muscle experiments, was widely adopted. However, this macro phenomenal model neglected the linkage to the microscopic characteristics of the skeletal muscle. At the micro level, Huxley proposed the sliding-filament theory which described that the muscle contraction resulted from of the relative slip between the thick and thin filaments, induced by myosin molecular motors. However, in his research, the description for the cycling working process of myosin motors is insufficient. Myosin motor is one kind of protein that transfers chemical energy from catalytic hydrolysis ATP (Adenosine Triphosphate) into mechanical work, i.e., directional movement [6-9]. The mechanochemical coupling of myosin motors is then used to understand the micro-mechanical behavior of skeletal muscle.

Lymn and Taylor first described myosin motor's work cycle, considering four steps, in 1971 [8,10]. Julicher et al. [11] then proposed a two-state brown ratchet model for a single motor and obtained the collective properties of molecular motors. This model has been extended to different families of molecular motors, such as kinesin, dynein, and myosin. Chin et al. [12] divided the biochemical cycle of myosin motor into seven states, and proposed a simulation model that allows altering the rates of transitions, mimicking the role of chemicals, including ATP, ADP, etc., between any of the states in a stochastic cycle. Wang [13] developed a mathematical model for investigating the mechanochemical coupling mechanism of molecular motors. However, these models lack the quantitative linkage to the bulk behaviors of muscle contractions.

Lecarpentier et al. [14] related the macro properties of skeletal muscle, such as maximum unloaded shortening velocity, peak force, the cross-sectional area of a muscle strip with the micro 
characteristics, including the force per single cross-bridge, probability distribution between each working state, and peak muscle efficiency. The statistical mechanics combined with Huxley's formula was used in their study.

The goal of this study is to develop a multiscale mathematical model for quantifying the mechanical behaviors of the active contraction skeletal muscle in relation with the mechanochemical coupling of molecular motors. First, the four states of the working mechanisms of myosin molecular motors in muscle contraction are analyzed. The evolution process of myosin molecular motors, regulated by load, chemicals, and Brownian force, was described. Second, the Fokker-Planck Equation was used to describe the collective behavior of myosin molecular motors. Third, the active contraction force and velocity of muscle fiber are calculated using the non-equilibrium statistical mechanics; and, finally, the biomechanical properties, at multiple scales, include probability density distribution of myosin motors and muscle fiber contraction force and velocity, are quantitatively related. Our results were then compared with published experiments and predicted models.

\section{Biomechanical Model of Skeletal Muscle Contraction}

The biomechanical model of skeletal muscle is generally characterized by parameters that reflect the physical structure of fiber (e.g., cross-sectional area, fiber length) and the behavior of molecular motors (e.g., transition rates between different chemical state, the free energy barriers established in the chemical reaction). The magnitudes of these parameters correspond to various fiber behaviors, such as fast and slow fibers. Regardless of these alternations, the mechanochemical coupling induced contraction force is the common feature among skeletal muscle.

\subsection{Working Mechanism of Myosin Molecular Motor}

In general, the muscle-contraction process consists of three steps: the muscle cell is excited, filaments slip and muscle contracts, and muscle stretches. Relative slip between the thick and thin filament is the direct cause of muscle contractions. The slip driving force is generated by the interaction between myosin motor and actin. The $\mathrm{Ca}^{2+}$ concentration in sarcomere increases suddenly under nerve impulse excitation, then, the binding sites on thin actin filaments are opened and the cross-bridge of myosin can blind with actin. Then, a power-stroke starts through the hydrolysis of ATP and causes the slipping between thin and thick filaments [4]. A large number of myosin motors acting on the thin filaments leads to the contraction of sarcomere.

The cycle of each myosin motor, first described by Lymn and Taylor [8,10], was divided into four main steps, also referred to as strokes, i.e., Detachment-stroke, when myosin motor detaches from actin with the new attachment of ATP; Recovery-stroke, when myosin moves forward to be ready for reattachment to the actin; Attachment-stroke, when myosin adheres to the actin; and Power stroke, when the myosin moves backward and forms a strong binding to the actin and the stored chemical energy released through ATP hydrolysis. One working cycle is completed by four chemical states, coupled with conformational changes, and the movement of a myosin motor, from one binding site to the next binding site, is then achieved. 


\subsection{Collective Dynamic Model of Myosin Molecular Motors in Sarcomere}

A myosin motor has many degrees of freedom associated with the unidirectional motion of the motor. The one-dimensional motion along the filament's slip direction is considered in this work. The distance between two binding sites on a thin filament is designated as $L$. A working cycle of a myosin motor is divided into $N=4$ steps, each step corresponding to one chemical state. $\sigma_{i}$ is used to represents the state $i$. Myosin motor transitions stochastically between different states, under the effect of existing external energy (for example, released from ATP hydrolysis), and a myosin motor can move directionally and push the thin filaments. Let $x$ be the variable as the displacement of a myosin motor along the thin filament, due the conformational change of the cross-bridge, is the relative slipping distance between thin and thick filaments. Let $\rho(x, t)$ be the probability density that the myosin motor, at the position of $x$ in the occupancy state at time $t$, possesses.

As the size and mass of molecular motors are relatively small, their stochastic motion follows the law of Brownian motion, which was well described by the Langevin Equation, regardless of inertia. In addition, the change of chemical state occupancy is depicted by a discrete Markov process. The combination of the two aforementioned governing equations then regulate the stochastic evolution of molecular motors.

For the molecular motor alone, the mechanochemical coupling mechanism is the function of multi-force interactions, including the motor force, the external load, the Brownian force obeying the fluctuation dissipation theorem, and the active force produced by chemical reactions. Its stochastic evolution of the probability density is represented by the Fokker-Planck Equation [14-16], as follows:

$$
\frac{\partial \rho_{i}}{\partial t}=D \frac{\partial}{\partial x}\left[\frac{1}{k_{B} T}\left(V_{i}^{\prime}(x)+F_{\text {Load }}\right) \rho_{i}\right]+D \frac{\partial^{2} \rho_{i}}{\partial x^{2}}+\sum_{j=1}^{N} k_{i j}(x) \rho_{j}
$$

where, $D=k_{\mathrm{B}} T / \gamma_{m}$, is the diffusion coefficient of myosin motor in aqueous solution, $k_{B}$ is the Boltzmann constant, $T$ is the absolute temperature, and $\gamma_{m}$ is a drag coefficient. The value $V_{i}(x)$ is the active potential energy produced by chemical reaction in state $i$, and is a function of the geometrical coordinate $x$ in the chemical occupancy state $i . F_{\text {Load }}$ is the external load force on the myosin motor and $k_{i j}(x)$ is the transition rate from chemical occupancy state $\sigma_{i}$ to state $\sigma_{j}$, the value depends on the potential energy in the two states. In addition, the probability density equation should satisfy the constraint defined by:

$$
\rho(0, t)=\rho(L, t)=0, \int_{0}^{L} \rho(x, t) \mathrm{d} x=1
$$

\subsection{Solving Fokker-Planck Equation}

The continuity equation (Equation (1)) is discretized as a spatially discrete Markov chain [16] to calculate the probability density distribution of myosin motors. A jump progress can then be used to estimate the continuous motion of myosin molecular motors, as demonstrated in Figure 1. The computational region $[0, L]$ is divided into $M$ sub-intervals, so the interval between two sites is $\Delta x=L / M$. Let $F_{i}^{n}$ and $B_{i}^{n}$ indicate the forward and backward jump rates between sites $x_{n-1}$ and $x_{n}$. The jump rates can be calculated as follows: 


$$
\begin{aligned}
\mathrm{F}_{i}^{n} & =\frac{D}{(\Delta x)^{2}} \frac{\Delta \phi_{i}^{n} / k_{B} T}{\exp \left(\Delta \phi_{i}^{n} / k_{B} T\right)-1} \\
\mathrm{~B}_{i}^{n} & =\frac{D}{(\Delta x)^{2}} \frac{-\Delta \phi_{i}^{n} / k_{B} T}{\exp \left(-\Delta \phi_{i}^{n} / k_{B} T\right)-1}
\end{aligned}
$$

where, $\Delta \phi_{i}^{n}=\phi_{i}\left(x_{n}\right)-\phi_{i}\left(x_{n-1}\right)$ and $\phi_{i}(x)=V_{i}(x)-x \cdot F_{\text {Load }}$.

The probability of the myosin motor between $\left[x_{n}, x_{n+1}\right]$ is calculated as:

$$
p_{i}^{n+1}=\int_{x_{n}}^{x_{n+1}} \rho_{i}(x, t) \mathrm{d} x
$$

Therefore, Equation (1) can be approximated by the master equation for the jump process:

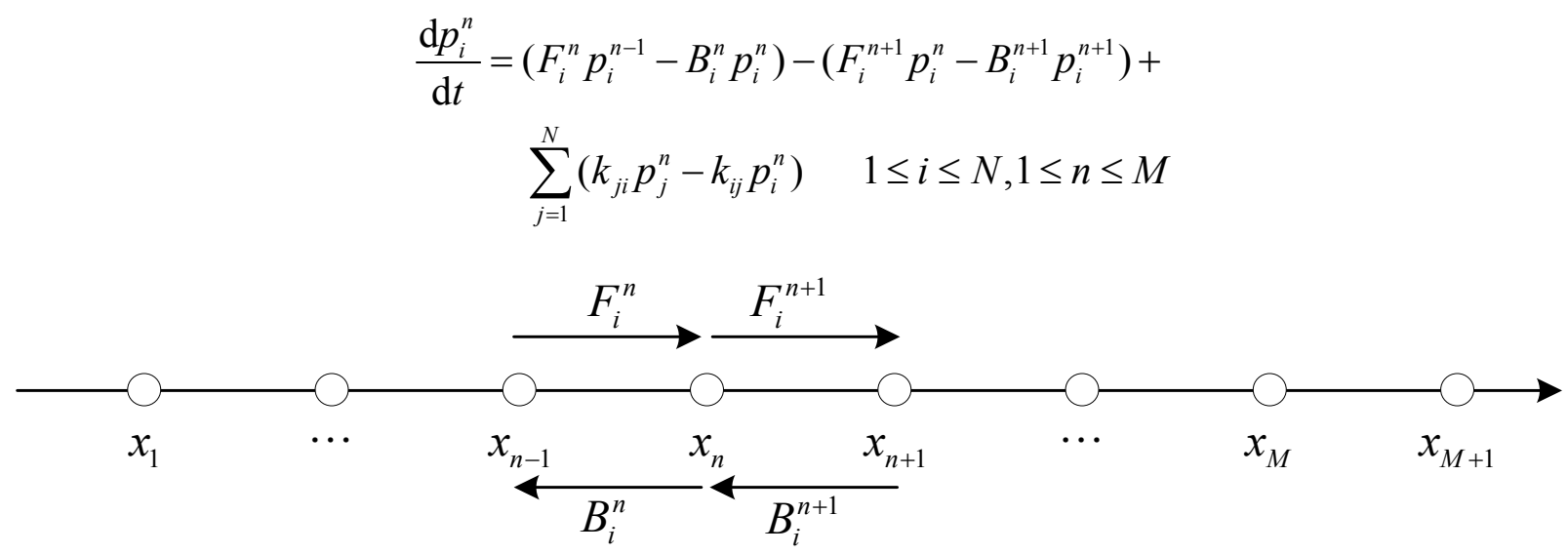

Figure 1. The spatial discretization and jump rates between two sites.

\subsection{Model Simplification}

It is challenging to solve the above equations due to the four steps in one complete working cycle of myosin motor. Considering that myosin motors only act in the case of a strong binding to the actin, thus, the working cycle of myosin motor can be simplified to two strokes: attachment-stroke and detachment-stroke, as depicted in Figure 2. Correspondingly, Equation (6) can be simplified as:

$$
\left\{\begin{array}{l}
\frac{\mathrm{d} p_{1}^{n}}{\mathrm{~d} t}=\left(F_{1}^{n-1} p_{1}^{n-1}-B_{1}^{n-1} p_{1}^{n}\right)-\left(F_{1}^{n} p_{1}^{n}-B_{1}^{n} p_{1}^{n+1}\right)+\left(k_{21} p_{2}^{n}-k_{12} p_{1}^{n}\right)=J_{1}^{n}-J_{1}^{n+1}+\left(k_{21} p_{2}^{n}-k_{12} p_{1}^{n}\right) \\
\frac{\mathrm{d} p_{2}^{n}}{\mathrm{~d} t}=\left(F_{2}^{n-1} p_{2}^{n-1}-B_{2}^{n-1} p_{2}^{n}\right)-\left(F_{2}^{n} p_{2}^{n}-B_{2}^{n} p_{2}^{n+1}\right)+\left(k_{21} p_{1}^{n}-k_{12} p_{2}^{n}\right)=J_{2}^{n}-J_{2}^{n+1}+\left(k_{21} p_{1}^{n}-k_{12} p_{2}^{n}\right)
\end{array}\right.
$$

where $p_{1}$ and $p_{2}$ are the probabilities in the two simplified states. The transition rates between the two states $\left(k_{12}\right.$ and $\left.k_{21}\right)$ are calculated as:

$$
\begin{gathered}
k_{12}=k_{-1} k_{-2} k_{-3} /\left(k_{-1}+k_{-2}+k_{-3}\right)+k_{4} \\
k_{21}=k_{1} k_{2} k_{3}[\mathrm{ATP}] /\left(k_{1}[\mathrm{ATP}]+k_{2}+k_{3}\right)+k_{-4}
\end{gathered}
$$

where $[\mathrm{ATP}]$ represents the concentration of ATP in the chemical reaction. The transition rate is influenced by the load force in the power stroke state, while it depends on free energy in other states. The relationship between transition rate and load force can be expressed as: 


$$
\begin{gathered}
k_{4}=k_{4}^{0} \exp \left(-\theta^{+} F_{\text {Load }} d / k_{B} T\right) \\
k_{-4}=k_{-4}^{0} \exp \left(-\theta^{-} F_{\text {Load }} d / k_{B} T\right)
\end{gathered}
$$

where $\theta^{+}$and $\theta^{-}$are the distribution coefficients that indicate the effect on the forward and backward transition rates, respectively [17]. $d$ is the moving distance of the molecular motor on the actin filament. The other transition rates $k_{i}=k_{i}^{0}(i=1,2,3)$ and $k_{-i}$ can be calculated with the detailed balance requirements [14].

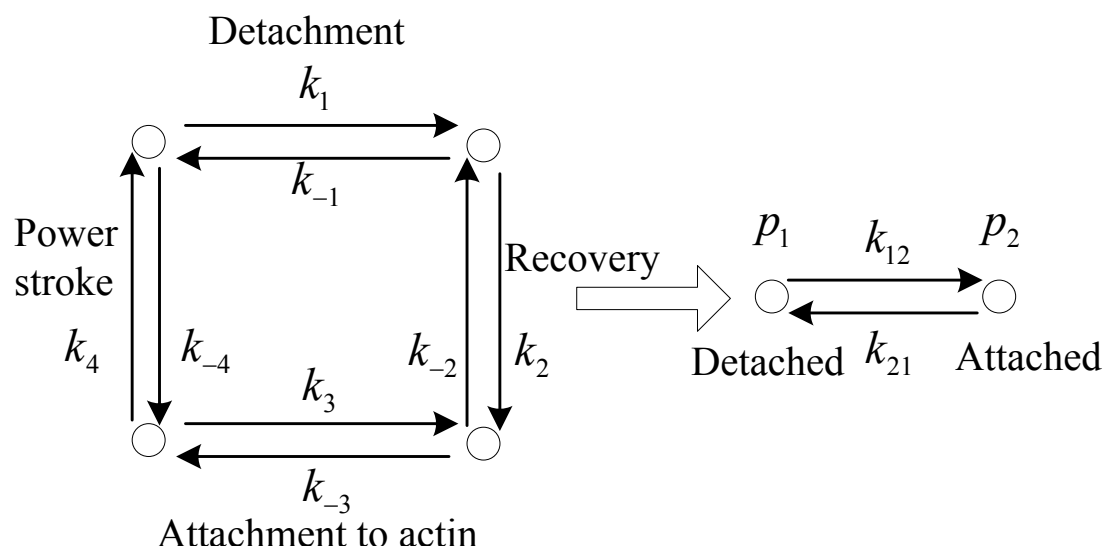

Figure 2. The simplification of working cycle of myosin motor.

Equation (7) could be written in a matrix form as:

$$
\frac{\mathrm{d}}{\mathrm{d} t}\left[\begin{array}{l}
\boldsymbol{p}_{1}^{n} \\
\boldsymbol{p}_{2}^{n}
\end{array}\right]=\left[\begin{array}{cc}
\boldsymbol{L}_{1}-k_{12} & k_{21} \\
k_{12} & \boldsymbol{L}_{1}-k_{21}
\end{array}\right]\left[\begin{array}{l}
\boldsymbol{p}_{1}^{n} \\
\boldsymbol{p}_{2}^{n}
\end{array}\right]
$$

The probability distribution subject to the constraints:

$$
\sum_{n=1}^{M} \sum_{i=1}^{N} \boldsymbol{p}_{i}^{n}=1
$$

where,

$$
\boldsymbol{L}_{i}=\left[\begin{array}{cccccc}
-\left(B_{i}^{1}+F_{i}^{2}\right) & B_{i}^{2} & & & & F_{i}^{1} \\
F_{i}^{2} & -\left(B_{i}^{2}+F_{i}^{3}\right) & B_{i}^{3} & & & \\
& F_{i}^{3} & -\left(B_{i}^{3}+F_{i}^{4}\right) & B_{i}^{4} & & \\
& & F_{i}^{4} & \ddots & \ddots & \\
& & & \ddots & \ddots & B_{i}^{M} \\
B_{i}^{1} & & & & F_{i}^{M} & -\left(B_{i}^{M}+F_{i}^{1}\right)
\end{array}\right], i=1,2
$$

The steady-state solution of the probability density distribution of molecular motors can be obtained by making the left side of Equation (12) equal to zero. In addition, according to the asymmetrical periodicity of potential, the two potentials are given by:

$$
\left\{\begin{array}{l}
V_{1}(x)=\Delta G\left[\sin \left(\frac{2 \pi x}{L}\right)-\frac{1}{2} \sin \left(\frac{4 \pi x}{L}\right)+\frac{1}{3} \sin \left(\frac{6 \pi x}{L}\right)\right] \\
V_{2}(x)=0
\end{array}\right.
$$


where $\Delta G$ indicates the energy derived from one ATP hydrolysis. The potentials determine the values of jump rates $F_{i}^{n}$ and $B_{i}{ }^{n}$ in Equation (12). The jump rates can be calculated with Equations (3) and (4). Equation (15) is used to represent the effect of the chemical reaction on the myosin motion, and link the chemical reaction and motor motion.

\subsection{Active Contraction Force and Velocity of Muscle Fiber}

Muscle fiber is composed of sarcomeres that are arranged in series and in parallel. According to mechanical principles, the muscle active contraction force is proportional to the number of sarcomeres in parallel. That is to say, the muscle active force is proportional to the cross-sectional area of muscle, once the force generated by each sarcomere is determined. In this work, we hypothesized that all sarcomeres' working processes are synchronized and that the $\mathrm{Ca}^{2+}$ concentration is large enough to detach all the binding sites on thin filaments during movement. Then, the probability distribution of myosin motors at any time can be used to calculate the magnitude of force acting on thin filament. The contraction force produced by all myosin motors in muscle fiber can be obtained as:

$$
F_{a}=\frac{\alpha A n_{0} k_{\mathrm{e}}}{s} \int_{0}^{L} x \rho(x, t) \mathrm{d} x
$$

where, $\int_{0}^{L} x \rho(x, t) \mathrm{d} x$ is the first-order of displacement, which also means the average displacement along the thin filament of all myosin motors, $n_{0}$ is the number of myosin motors in one thick filament, $k_{\mathrm{e}}$ is the elasticity of cross-bridge, $A$ is the cross-sectional area of muscle fiber, $s$ is the area of hexagonal surrounded by 6 thin filaments around a thick filament, $\alpha$ is the degree of overlap between thin and thick filaments, and as the sliding-filament theory confirms, there exists an optimal overlap to generate a maximum force [5], a Gaussian function is used to imitate the process in this paper:

$$
\alpha=\mathrm{e}^{-\left(l-l_{\text {opti }}\right)^{2} / 2 \mathrm{c}^{2}}
$$

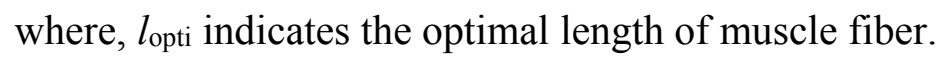

The muscle fiber contraction velocity of the sarcomere can be calculated by the evolution of the molecular motor as:

$$
v=\frac{1}{N} \sum_{i=1}^{N} L J_{i}
$$

where:

$$
\begin{gathered}
J_{i}=\frac{D}{k_{B} T}\left(V_{i}^{\prime}(x)+F_{\text {Load }}\right) \rho_{i}+D \frac{\partial \rho_{i}}{\partial x}=\sum_{n=1}^{M} J_{i}^{n} \\
v_{i}=L J_{i}
\end{gathered}
$$

where the probability flux and average drift velocity of molecular motor in state $i$ was defined through Equations (1) and (7) [16]. For a muscle fiber that is composed of sarcomeres, the contraction velocity will be:

$$
V=m_{s} v
$$

where, $m_{s}$ is the number of sarcomere that one myofibril contains in series. 


\section{Results and Discussions}

\subsection{Modeling Input Parameters}

A multiscale mathematical model of muscle contraction has been presented in the previous section, which required a few input parameters, including the physical structure of fiber and the operating conditions of molecular motors. We used the fast skeletal muscle fiber as the model system in this work. Lecarpentier [14] measured the fast muscle extensor digitalis longus (EDL) of rats, which shows that, for an EDL muscle, whose initial length is $15 \mathrm{~mm}$, the maximum isometric force is $85.1 \mathrm{mN} / \mathrm{mm}^{2}$, the max contraction velocity is $81.2 \mathrm{~cm} / \mathrm{s}$, and the area of the hexagon shape of a thick filament and six thin filaments is $1.375 \times 10^{3} \mathrm{~nm}^{2}$. In addition, the distance between two actin sites is $36 \mathrm{~nm}$, and the overall free-energy change after the hydrolysis of one ATP at normal cellular conditions is about $25 k_{B} T$. The input parameters for the fast muscle are summarized in Table 1. All the kinetic equations were then solved using MATLAB.

Table 1. Key parameters used in the model.

\begin{tabular}{cccc}
\hline $\boldsymbol{k}_{\boldsymbol{B}} \boldsymbol{T}$ & $\boldsymbol{D}$ & $\boldsymbol{S}$ & $\boldsymbol{L}$ \\
\hline $4.2 \mathrm{pN} / \mathrm{nm}$ & $5.47 \times 10^{7} \mathrm{~nm}^{2} / \mathrm{s}$ & $1.375 \times 10^{3} \mathrm{~nm}^{2}$ & $36 \mathrm{~nm}$ \\
$K_{\mathrm{e}}$ & $n_{0}$ & $M$ & $d$ \\
$0.65 \mathrm{pN} / \mathrm{nm}$ & 150 & 200 & $10 \mathrm{~nm}$ \\
\hline
\end{tabular}

\subsection{Probability Density Distribution}

Our results, shown in Figure 3, have demonstrated that the probability density distribution varied with the applied loading F, transition rates depending on [ATP], and motor potential profile. It is clear that the probability density of myosin motors at the attachment state, corresponding to the power-stroke state in the four-step working cycle, increased with higher loading. This indicates that the motor need to generate lager constrain force for overcoming the external loading. Considering different ATP concentrations, the probability density also increases with [ATP], but the speed of this change was reduced with a larger [ATP]. It reached a saturation point when [ATP] is 30 micromolar $(\mu \mathrm{M})$, which suggests that the active contraction force reached a maximum. Our results agree with the experimental observations by Cooke et al. [18]. They have shown that isometric tension and contraction velocity of rabbit psoas fiber increases to a maximum when ATP concentration reaches an optimal value at approximately $30 \mu \mathrm{M}$. Figure $3 \mathrm{c}$ demonstrates that the free energy $\Delta \mathrm{G}$ released from a single ATP might not alter the probability of myosin motors in a combined state, but it altered the potential profile. Specifically, the probability of myosin motors in the combined state remained at 1 , in which case the probability in the detached state could be $p_{2}=0.659$, complemented to $p_{1}=0.341$. However, with the increase of free energy released from ATP, the motors obtain a shorter average displacement along the thin filament. The explanation is that the required force for a certain number of motors is achieved with a smaller displacement as more chemical energy was transformed. 


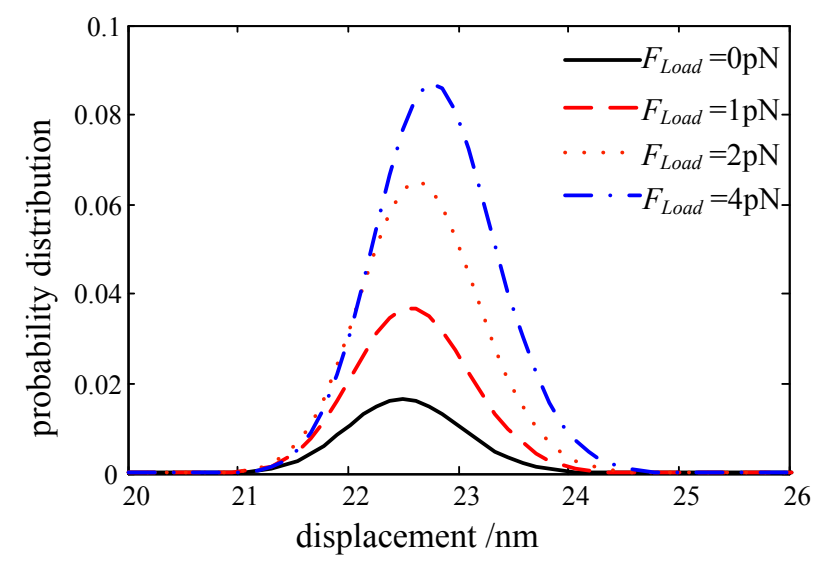

(a)

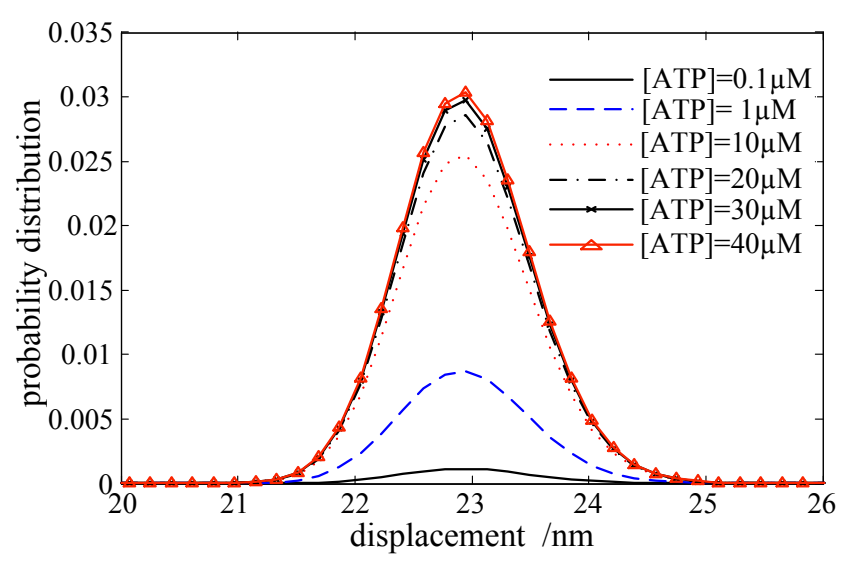

(b)

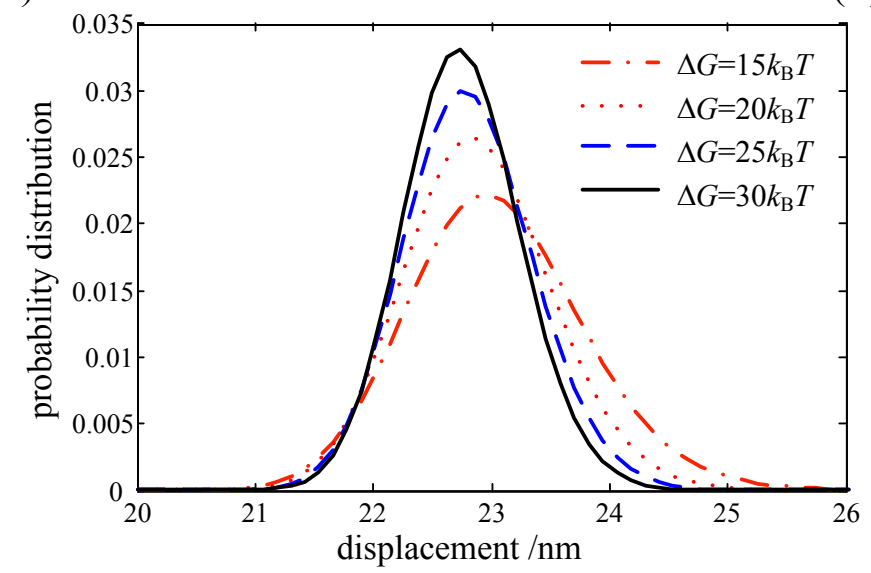

(c)

Figure 3. Probability density distribution of the molecular motor in relation to (a) various loads with $[\mathrm{ATP}]=30$ micromolar; $(\mathbf{b})$ ATP concentration with load $F_{\text {Load }}=6 \mathrm{pN}$; and (c) potential profiles with $F_{\text {Load }}=6 \mathrm{pN}$.

\subsection{Active Contraction Force and Velocity}

The active contraction force, according to Equation (16), was affected by the displacement of motors, the amount of thick filaments in one muscle fiber, the number of myosin motors in one thick filament, the elasticity of the cross-bridge, and the degree of overlap between thin and thick filaments. Considering a muscle fiber with a cross-sectional area of $1 \mathrm{~mm}^{2}$ and a length of $15 \mathrm{~mm}$, measured by Lecarpentier [14], its active contraction force and velocity were then calculated.

It was clearly illustrated in Figure 4a that, the active contraction force increased quickly with the ATP concentration when the ATP concentration is less than $30 \mu \mathrm{M}$. Afterwards, the active contraction force remained the same, regardless of the ATP concentration. This is consistent with Figure 3b. With the overlap coefficient $\alpha=1$, the load force on one sarcomere is estimated as $30 \mathrm{pN}$, i.e., $2 \mathrm{pN}$ on each motor. One single thick filament could then generate $107.1 \mathrm{pN}$ considering that there are $7.3 \times 10^{8}$ thick filaments arranged in parallel in muscle fiber per $1 \mathrm{~mm}^{2}$. The maximum active force induced by the muscle fiber is calculated as $78 \mathrm{mN}$, which is within $10 \%$ variation from the measured maximum isometric forces of $85.1 \mathrm{mN} / \mathrm{mm}^{2}$ [14]. The difference could be minimized by adjusting the amount of motors in one sarcomere and/or its elasticity, which are adopted from different resources $[4,14]$. Figure $4 \mathrm{~b}$ depicted the relationship between active contraction force and fiber length obtained from 
Equation (17), where the maximum contraction force of $80 \mathrm{mN}, 60 \mathrm{mN}$, and $40 \mathrm{mN}$ corresponded to the muscle fiber's activation level at $100 \%, 75 \%$, and $50 \%$, respectively. It is clear that the muscle active force reached its peak state at the optimal fiber length. This result agrees with the prediction from Hill's model [2,5], as shown in Figure 4c.

Figure 5 demonstrated the relationship between sarcomere contraction velocity and load force on sarcomere under different ATP concentrations, calculated from Equations (18)-(21). The obtained velocity-force (V-F) curve, normalized by the maximum velocity and maximum load force, respectively, is an important characteristic in muscle contraction. Equations have shown that the muscle contraction velocity is proportional to the average drift velocity of the myosin motor, which is then related to load force per motor. Our results further quantified that the contraction velocity decreased with a larger force and slowed to zero, as the force is too large for the myosin to drive. Our observations are consistent with the V-F curve fitted from experiments, referred to as V-F of Hill in Figure $5[2,12]$.

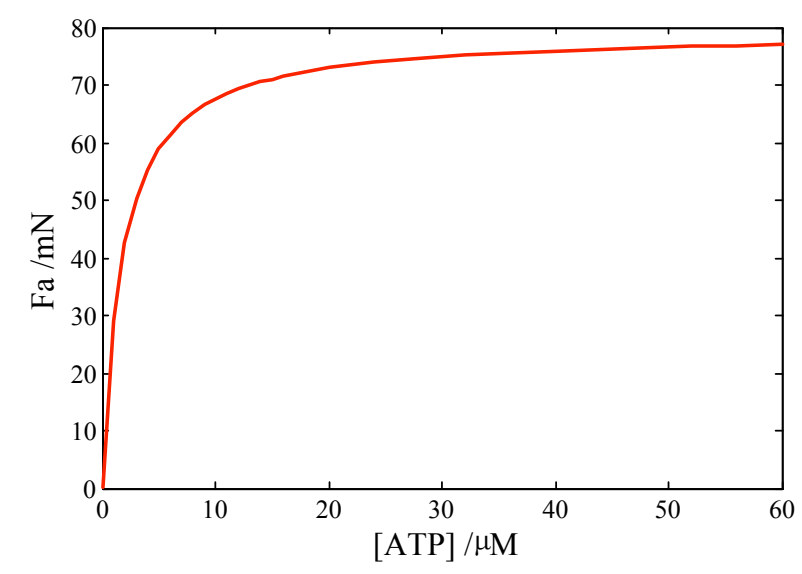

(a)

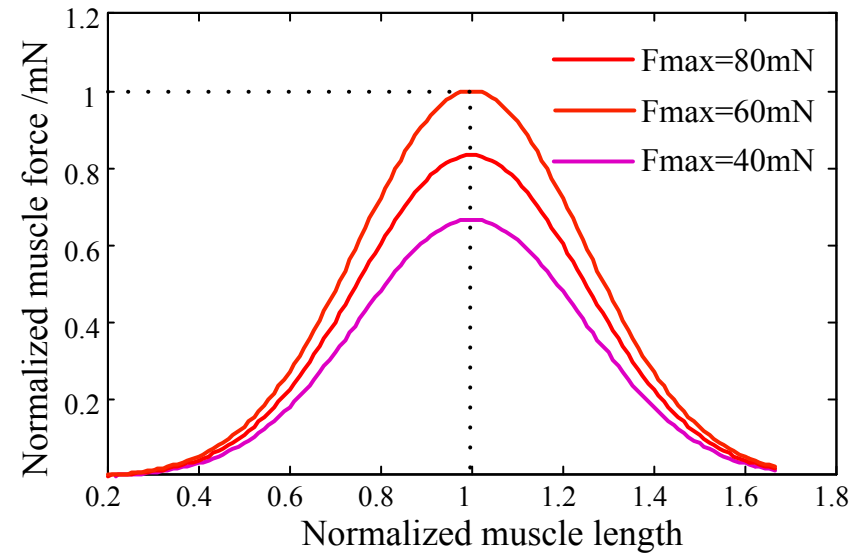

(b)

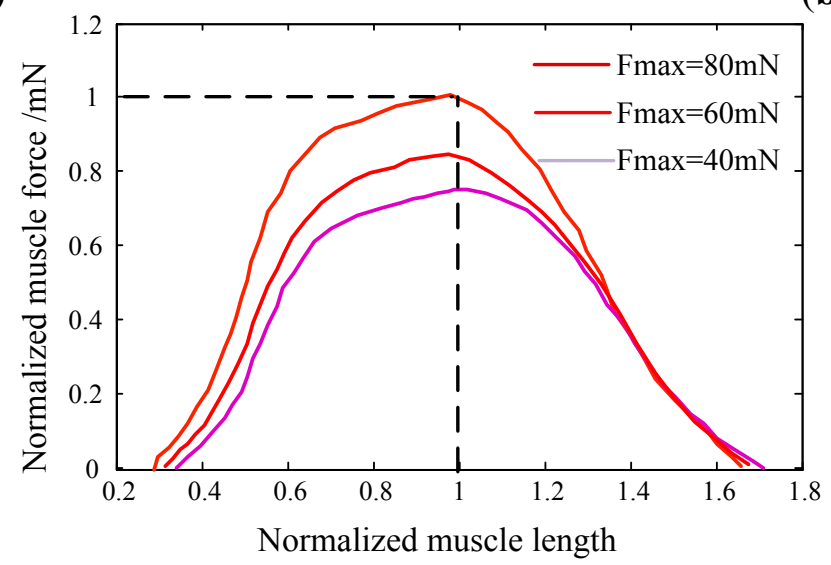

(c)

Figure 4. (a) The relationship between active contraction force and [ATP]; Active force-length curves, normalized by the maximum muscle force and optimal fiber length, respectively, calculated from either our model (b) or the Hill model (c). 


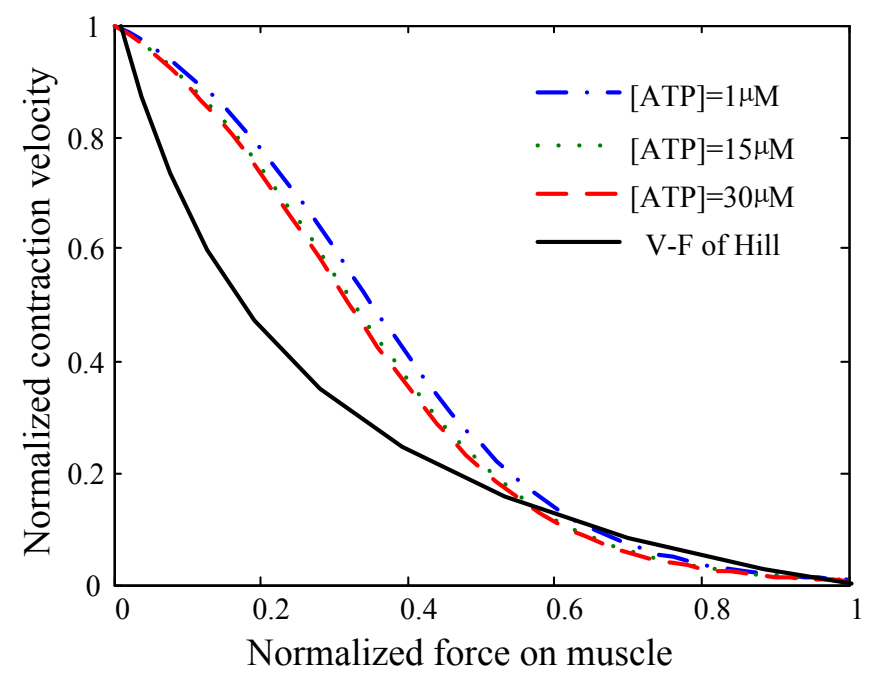

Figure 5. The normalized V-F curves.

\section{Conclusions}

In this work, a collective dynamic model of myosin molecular motors is developed, based on both the Langevin Equation and Markov process. Its behaviors are then coupled with the active contraction force and contraction velocity of skeletal muscle at the macroscopic scale. The roles of motor potential profile, ATP concentration, and load force on myosin motors are investigated Results are summarized as: (i) The probability of the attachment state in the two-step working cycle increases with a larger load force of motors; (ii) the probability density in the attachment state increases with [ATP] but reaches a saturation state at a certain [ATP]; (iii) the obtained force-length and velocity-force properties of muscle are validated against experimental observations. This indicated that our multiscale model could capture the major features of muscle contractions; and (iv) the proposed multiscale model in this work could be used for characterizing any muscle contraction.

In the present model, the force-length relationship was assumed as symmetric regarding optimal muscle length. Its asymmetry could be implemented in the future work using a modified Gaussian function. The sarcomere was also assumed to be fully activated, i.e., the $\mathrm{Ca}^{2+}$ concentration is high enough for myosin motors to bind strongly with the actin. However, the $\mathrm{Ca}^{2+}$ concentration is regulated by the firing rate of nerve impulses. Despite these simplifications, the present work demonstrated the importance of multiscale modeling for a better understanding the mechanism of skeletal muscle active contractions. Quantitative relation between myosin molecular motors and muscle contractions could be used to provide guidance for designing effective rehabilitation exercises, improving performance in competitive sports, and exploiting the potential to prevent muscle injuries.

\section{Acknowledgments}

The authors are very grateful for the research supported by the National Natural Science Fund (No. 91420301). 


\section{Author Contributions}

Jiangcheng Chen and Xiaodong Zhang conceived and designed the study; He Wang performed the simulation; Linxia Gu and Shengmao Lin critically revised the manuscript.

\section{Conflicts of Interest}

The authors declare no conflicts of interest.

\section{References}

1. Nordin, M.; Frankel, V. Basic Biomechanics of the Musculoskeletal System, 4th ed.; Lippincott Williams \& Wilkins: Philadelphia, PA, USA, 2012; pp. 148-175.

2. Hill, A.V. The heat of shortening and the dynamic constants of muscle. Proc. R. Soc. Lond. Ser. B Biol. Sci. 1938, 126, 136-195.

3. Huxley, A.F. Muscle structure and theories of contraction. Prog. Biophys. Biophys. Chem. 1957, 7, 255-318.

4. Lan, G.; Sun, S.X. Dynamics of myosin-driven skeletal muscle contraction: I. Steady-state force generation. Biophys. J. 2005, 88, 4107-4117.

5. Lloyd, D.G.; Besier, T.F. An EMG-driven musculoskeletal model to estimate muscle forces and knee joint moments in vivo. J. Biomech. 2003, 36, 765-776.

6. Maclntosh, B.R.; Gardiner, P.F.; McComas, A.J. Skeletal Muscle: Form and Function, 2nd ed.; Human Kinetics Publishers, Inc.: Champaign, IL, USA, 2006.

7. Campas, O.; Kafri, Y.; Zeldovich, K.B.; Casademunt, J.; Joanny, J.-F. Collective dynamics of interacting molecular motors. Phys. Rev. Lett. 2006, 97, 038101.

8. Lymn, W.R.; Taylor, E.W. Mechanism of adenosine triphosphate hydrolysis by actomyosin. Biochemistry 1971, 10, 4617-4624.

9. Vermeulen, K.C.; Stienen, G.J.; Schmidt, C.F. Cooperative behavior of molecular motors. J. Muscle Res. Cell Motil. 2002, 23, 71-79.

10. Fischer, S.; Windshügel, B.; Horak, D.; Holmes, K.C.; Smith, J.C. Structural mechanism of the recovery stroke in the myosin molecular motor. Proc. Natl. Acad. Sci. USA 2005, 102, 6873-6878.

11. Julicher, F.; Ajdari, A.; Prost, J. Modeling molecular motors. Rev. Mod. Phys. 1997, 69, 1269-1281.

12. Chin, L.; Yue, P.; Feng, J.J.; Seow, C.Y. Mathematical simulation of muscle cross-bridge cycle and force-velocity relationship. Biophys. J. 2006, 91, 3653-3663.

13. Wang, H. Chemical and mechanical efficiencies of molecular motors and implications for motor mechanisms. J. Phys. Condens. Matter 2005, 17, S3997.

14. Lecarpentier, Y.; Blanc, F.-X.; Quillard, J.; Hébert, J.-L.; Krokidis, X.; Coirault, C. Statistical mechanics of myosin molecular motors in skeletal muscles. J. Theor. Boil. 2005, 235, 381-392.

15. Xing, J.; Wang, H.; Oster, G. From continuum Fokker-Planck models to discrete kinetic models. Biophys. J. 2005, 89, 1551-1563.

16. Wang, H.; Charles, S.P.; Timothy, C.E. A robust numerical algorithm for studying biomolecular transport processes. J. Theor. Boil. 2003, 221, 491-511. 
17. Zhang, Y. A general two-cycle network model of molecular motors. Phys. A Stat. Mech. Appl. 2009, 388, 3465-3474.

18. Cooke, R.; Bialek, W. Contraction of glycerinated muscle fibers as a function of the ATP concentration. Biopgysical J. 1979, 28, 241-258.

(C) 2015 by the authors; licensee MDPI, Basel, Switzerland. This article is an open access article distributed under the terms and conditions of the Creative Commons Attribution license (http://creativecommons.org/licenses/by/4.0/). 